ARTICLE

Received 26 Sep 2016 | Accepted 7 Apr 2017 | Published 30 May 2017

DOI: $10.1038 /$ ncomms15550 OPEN

\title{
Hydrodynamic cavitation in Stokes flow of anisotropic fluids
}

\author{
Tillmann Stieger ${ }^{1}$, Hakam Agha ${ }^{2,3}$, Martin Schoen ${ }^{1,4}$, Marco G. Mazza² \& Anupam Sengupta 5,6
}

Cavitation, the nucleation of vapour in liquids, is ubiquitous in fluid dynamics, and is often implicated in a myriad of industrial and biomedical applications. Although extensively studied in isotropic liquids, corresponding investigations in anisotropic liquids are largely lacking. Here, by combining liquid crystal microfluidic experiments, nonequilibrium molecular dynamics simulations and theoretical arguments, we report flow-induced cavitation in an anisotropic fluid. The cavitation domain nucleates due to sudden pressure drop upon flow past a cylindrical obstacle within a microchannel. For an anisotropic fluid, the inception and growth of the cavitation domain ensued in the Stokes regime, while no cavitation was observed in isotropic liquids flowing under similar hydrodynamic parameters. Using simulations we identify a critical value of the Reynolds number for cavitation inception that scales inversely with the order parameter of the fluid. Strikingly, the critical Reynolds number for anisotropic fluids can be $50 \%$ lower than that of isotropic fluids.

\footnotetext{
${ }^{1}$ Stranski-Laboratorium für Physikalische und Theoretische Chemie, Technische Universität Berlin, Straße des 17. Juni 115, 10623 Berlin, Germany. ${ }^{2}$ Max Planck Institute for Dynamics and Self-Organization (MPIDS), Am Faßberg 17, 37077 Göttingen, Germany. ${ }^{3}$ Physics and Material Science Unit, University of Luxembourg, 162 Avenue de la Faiencerie, L-1511 Luxembourg, Luxembourg. ${ }^{4}$ Department of Chemical and Biomolecular Engineering, North Carolina State University, Engineering Building I, Box 7905, 911 Partners Way, Raleigh, North Carolina 27695, USA. ${ }^{5}$ Ralph M. Parsons Laboratory for Environmental Science and Engineering, Department of Civil and Environmental Science and Engineering, Massachusetts Institute of Technology, 15 Vassar Street, Cambridge, Massachusetts 02139, USA. ${ }^{6}$ Institute for Environmental Engineering, Department of Civil, Environmental and Geomatic Engineering, ETH Zurich, StefanoFranscini-Platz 5, 8093 Zurich, Switzerland. Correspondence and requests for materials should be addressed to M.G.M. (email: marco.mazza@ds.mpg.de) or to A.S. (email: anupams@ethz.ch).
} 
C avitation, the nucleation of vapour bubbles within a liquid, is one of the oldest and most extensively researched phenomena in fluid dynamics ${ }^{1-4}$. Commonly triggered by local heating of a fluid above its boiling temperature (superheating), or by physical processes that abruptly decrease the fluid's pressure below its saturated vapour pressure ${ }^{5}$, cavitation is often accompanied by pernicious ramifications. The drop in fluid pressure during cavitation can have considerable implications on a range of industrial and biomedical processes. Industries conventionally engaged in tribology 6,7 and hydraulics of mechanical components ${ }^{8,9}$, sonochemical ${ }^{10,11}$ and cryogenic processing ${ }^{12}$ and high-throughput extrusion processes in polymer material plants ${ }^{13}$ routinely experience material degradation due to cavitation-induced wear and tear, resulting in considerable economic losses. The scope and the extent to which cavitation underpins crucial steps in biophysical and biomedical processes have recently started to be recognized. Whether it is ascent of the sap in tall trees ${ }^{14}$, the lubrication efficacy of thin films of synovial fluids between our joints ${ }^{15}$, the performance of a mechanical heart valve ${ }^{16}$ or the ability of shrimps to deftly employ it as a tool to stun their preys ${ }^{17}$, cavitation is the common functional determinant in a range of biological processes.

Cavitation sets in when liquids are subject to tensile stresses. Above a critical value, known as the breaking tension or cavitation threshold ${ }^{18}$, liquids experience negative pressures, a thermodynamically metastable state ${ }^{19,20}$. While in some cases reaching the cavitation threshold may be the desired goal (for example, in applications based on sonomechanics), in others, the physical and material parameters are tuned to avoid the inception of cavitation. Hydrodynamic cavitation-the cavitation in liquids induced by a hydrodynamic flow-has been the subject of both experiments and extensive simulations, including molecular dynamics (MD) simulations of LennardJones fluids ${ }^{21,22}$, Monte Carlo simulations ${ }^{23-25}$ and density functional theory ${ }^{26-29}$. From inception to implosion, the life time of a cavitating bubble can depend on a number of factors: volume of dissolved gases in the liquid matrix, presence of inclusions (for example, particulate matters) or pre-existing nucleation sites (for example, gas bubbles) and roughness of the solid surfaces in contact with the liquid. Such multiparameter dependence can pose technical challenges to study cavitation experimentally $-\mathrm{a}$ possible explanation for the lack of corresponding investigations in anisotropic liquids.

Anisotropic liquids constitute a special class of complex, non-Newtonian liquids, in which the molecules exhibit long-range order in their orientations or positions. Common examples of such liquids include liquid crystals (LCs) ${ }^{30,31}$, polymeric liquids far from equilibrium (LC polymers) ${ }^{32}$ and electro/magnetorheological fluids. In such partially ordered media, the hydrodynamic properties, and outcomes, are determined not just by the inertial and viscous parameters, but also by the long-range elastic interactions between molecules, and those between the molecules and confining boundaries. Predictors of cavitation in polymeric and ordered liquids have been subject of a few simulation-based studies, including MD simulations of the nucleation of cavities in a homogeneous polymer under tensile strain ${ }^{33}$, and within polymers containing nanocomposites ${ }^{34}$. Interestingly, a number of building blocks in living organisms are anisotropic ${ }^{35-38}$ : amphiphilic lipids in cellular membranes, cytoskeletal and muscle proteins and the collagens and proteoglycans of connective tissues possess intrinsic order; and biopolymer solutions in the cytoskeleton ${ }^{39}$ and F-actin filaments ${ }^{40,41}$ have nematic symmetry. Consequently, the study of cavitation in complex anisotropic media becomes essential, especially in light of biomedical procedures like electrohydraulic lithotripsy ${ }^{42}$, and shock-wave lithotripsy that can trigger intraluminal bubble expansion leading to rupture of capillaries and small blood vessels ${ }^{43}$.

Despite its far-reaching implications, a systematic study of cavitation in anisotropic liquids is yet to be undertaken. LCs and LC polymer materials, with their highly tunable physical properties ${ }^{44}$, offer a realistic substitute for biological samples to carry out investigations of cavitation within anisotropic media. To the best of our knowledge, cavitation in LC materials has been studied only in the contexts of phase transitions in LCs under negative pressure ${ }^{45}$, and the disruption of director alignment ${ }^{46}$. While in the former case, cavitation was induced by an isochoric cooling of small LC droplets embedded in a glass-forming material, acoustic waves were employed in the latter to nucleate cavitating domains. Any attempt to study hydrodynamic cavitation in LCs is still largely lacking.

In this work, we use microfluidic experiments to study hydrodynamic cavitation in a liquid crystalline material (5CB, nematic phase at room temperature, see Methods), and employ MD simulations to quantitatively capture the dynamics of cavitation inception and growth. Cavitation occurred due to the flow of nematic 5CB past a cylindrical pillar, placed within a linear microfluidic channel. A vapour-liquid interface, pinned on the micropillar surface, encases the cavitation domain. Interestingly, the inception and growth of the domain takes place in the Stokes flow regime (Reynolds number, Re $\ll 1$ ), that is, under laminar flow conditions. We have identified a range of Ericksen numbers, $200<\mathrm{Er}<500$ (the Ericksen number characterizes the nematofluidic conditions of our system ${ }^{44}$, see Methods), in which cavitation is reproducible and stably sustained. From its first appearance at a given Ericksen number, the cavitation domain grows in size, and within a few hours, attains the saturation volume. For Ericksen numbers below the lower bound, no cavitation was observed, whereas at $\mathrm{Er}>500$, the domain was unstable, and was observed to shrink in size. In all our experiments, the cavitation volume was localized around the stagnation point downstream of the micropillar. Using MD simulations, we numerically reproduce the microfluidic experiments and, by identifying the material and flow parameters, establish the physical principles that govern cavitation in anisotropic liquids. We find that the critical Reynolds number, $\operatorname{Re}_{\text {cr }}$ (minimum Re for cavitation inception), scales inversely with the order parameter of the anisotropic liquid. This, in contrast to corresponding flows in isotropic phase (order parameter $\simeq 0$ ), was calculated to be less than half of that in isotropic liquids. Finally, we develop theoretical arguments based on the free energy of the fluid that support all our findings.

\section{Results}

Experiments. The first appearance of the microscopic cavitation domain was detected at $\mathrm{Er} \simeq 200$. As shown in Fig. 1a,b, the vapour phase localizes at the hydrodynamic stagnation point downstream of the micron-sized pillar. As the streamlines of the flowing 5CB divide upon approaching the micropillar, both $\mathrm{Er}$ and $\mathrm{Re}$ first increase locally, reach maximum values at the point of minimum separation and reattain their original values as the flows converge downstream of the pillar. With an average flow speed of $800 \mu \mathrm{ms}^{-1}(\mathrm{Er}=200)$, the velocity reaches a maximum of $4,000 \mu \mathrm{ms}^{-1}$ at the constriction (minimum distance of $10 \mu \mathrm{m}$ between the pillar surface and the channel wall). This corresponds to $\mathrm{Er}=945$ and $\mathrm{Re}=0.1$, a hydrodynamic Stokes regime. The resulting drop in the hydrodynamic pressure corresponds to $\simeq 8 \mathrm{mPa}$, corroborating that the absolute pressure decreases below the vapour pressure of nematic $5 \mathrm{CB}\left(\simeq 0.1 \mathrm{mPa}\right.$ at $\left.298 \mathrm{~K}^{47}\right)$. 

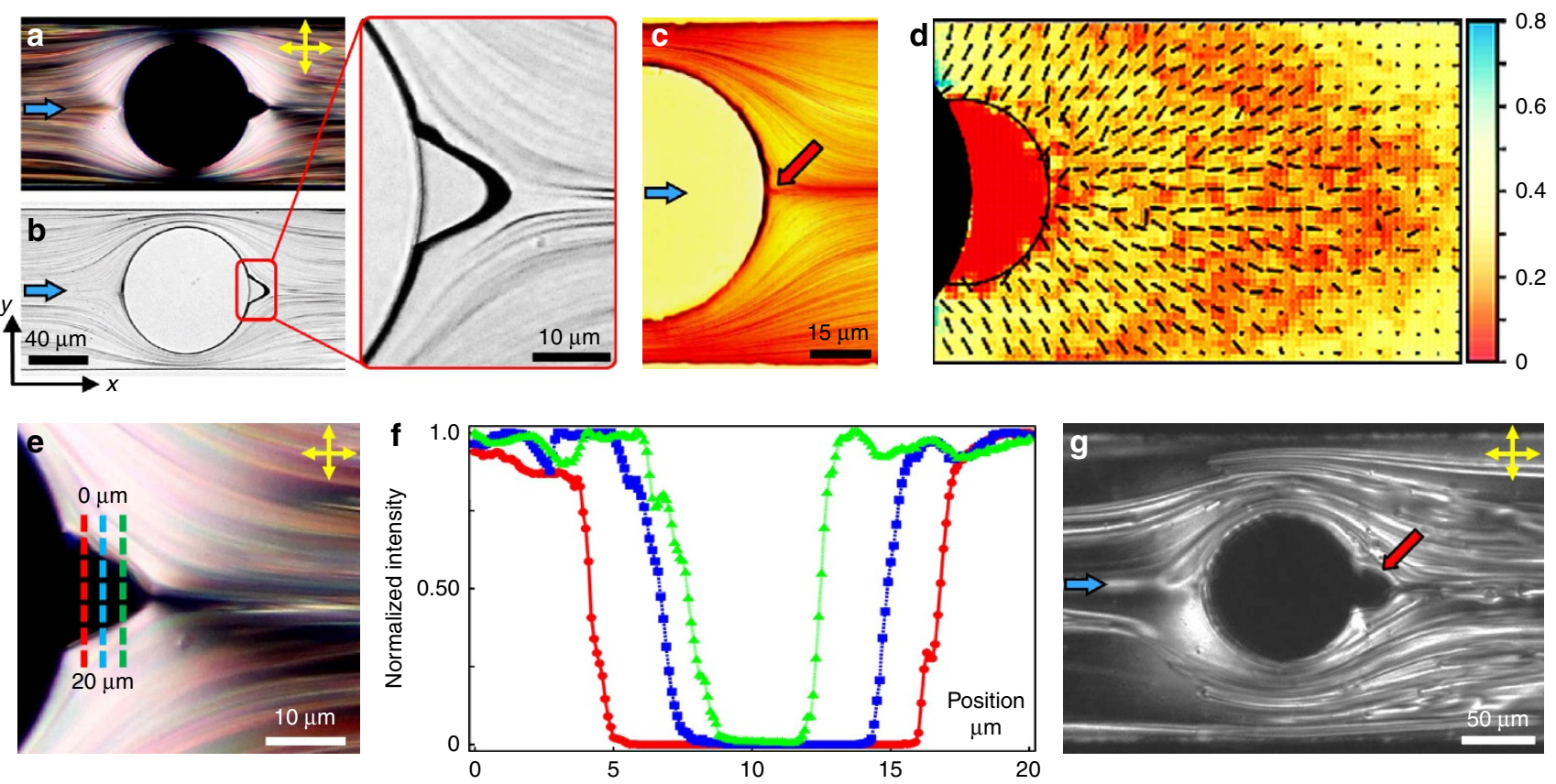

Figure 1 | Cavitation in nematic LC due to flow past a micron-sized pillar. (a) Polarized optical micrograph and (b) white light micrograph show steady-state cavitation domain in nematic LC, 5CB. The channel and pillar surfaces have homeotropic boundary condition. Right panel: Magnified projection of the cavitating domain. (c) Minimum intensity projection of the scattered light over time due to flowing disclination lines. The disclinations were used to trace the streamlines of the LC flow past the micropillar. The cavitating domain is locally stabilized at the hydrodynamic stagnation point (indicated by the red arrowhead) downstream of the micron-sized pillar. For the micrographs shown here, the pillar diameter is $2 r=80 \mu \mathrm{m}$, placed within a $100 \mu \mathrm{m}$ wide and $15 \mu \mathrm{m}$ deep microchannel. (d) Map of the local nematic order parameter in proximity of the cavitation domain obtained from MD simulations (see attached colour bar). The area of solid red colour corresponding to vanishing nematic order represents the cavitation. Small dashes in the maps represent the local director field. The length of a dash represents its three-dimensional orientation: shorter dashes have an orientation closer to the normal to the plane of the map. (e) Magnified view of the cavitating domain observed between crossed polarizers. The intensity of the transmitted light (normalized by the maximum intensity) is measured along the dashed lines: red, blue and green, and plotted in (f). For each line, the maximum value of the normalized intensity (bright regions) is recorded outside the cavitation domain (bulk director is oriented at $45^{\circ}$ relative to the polarizers). At the apex of the cavitation domain and further downstream, the director is oriented parallel to the flow direction that appears dark between the crossed polarizers. The gas-filled cavitation domain also appears dark (minimum intensity) due to the total extinction of transmitted light. (g) Cavitation domain was also observed under planar surface anchoring conditions, visualized here between crossed polarizers.

Figure 1c shows the downstream hydrodynamic stagnation point (red arrowhead), visualized using a differential intensity projection of the light scattered by the flowing disclinations (Methods). Due to the high Er flows, singular topological defects readily fills up the nematic bulk that scatter white light due to the lensing effect ${ }^{48}$. These freely flowing disclinations (both $\pm 1 / 2$ and \pm 1 defects are present) follow the streamlines, and accurately map the laminar flow field around the micropillar. Between the crossed polarizers, the cavitation domain has a dark appearance due to complete extinction of the transmitted white light. As shown in Fig. 1b, the interface between the vapour and liquid phases of $5 \mathrm{CB}$ is distinctly visible under white light. Cavitation was also observed within microfluidic devices possessing planar surface anchoring (Fig. 1g). Irrespective of the surface anchoring, no cavitation was observed in nematic flows at $\mathrm{Er}<200$. In all cases, the nematic director field, average orientation of the LC molecules, visualized using polarization microscopy, was observed to be aligned by the flow field (nematic 5CB is a flow aligning $\mathrm{LC}^{32}$ ). This agrees very well with flow-induced director field described previously ${ }^{49}$.

Figure 1d maps the local nematic order in proximity of the cavitation domain, obtained through MD simulations. In agreement with the polarized optical measurements (Fig. 1e), our simulations predict that the LC molecules anchor homeotropically at the fluid-vapour interface. As shown in Fig. 1f, the normalized intensity of the transmitted light, plotted for three adjacent sections on the cavitation domain (red, blue and green dashed lines), attains maximum value just outside the cavitation domain. The maximum intensity corresponds to bulk director orientation of $45^{\circ}$ relative to the crossed polarizers. Close to the apex of the cavitation domain, the director is oriented along the flow direction. This appears dark between crossed polarizers as the director is parallel to one of the polarizers. A minimum intensity is also observed inside the cavitation domain due to total extinction of the white light passing through the gas phase between crossed polarizers. The intermediate values of the intensity at the cavitation interface suggest that the molecules have a weak homeotropic alignment at the interface, as also confirmed by our MD simulations. Measurements on $5 \mathrm{CB}$ show that the molecules align homeotropically at the fluid-vapour interface ${ }^{50-52}$, in agreement with existing studies of the $\mathrm{LC}$-air interface for $5 \mathrm{CB}^{53}$. Our MD simulations correctly capture this behaviour that lends confidence to the predictions about the underlying physics. The disclination lines found in the experiments are not present in the MD system (Fig. 1d) as they require much larger system sizes to develop.

Upon appearance, the cavitation volume increases over time, and typically within few hours (4-7 h) of steady flow of the nematic $5 \mathrm{CB}$, saturates into a constant size. Figure 2a,b show polarization optical microscopy (POM) micrographs of a cavitating domain developing over $5 \mathrm{~h}$ at $\mathrm{Er} \simeq 210$. The time taken to attain the steady-state volume varies in experiments 


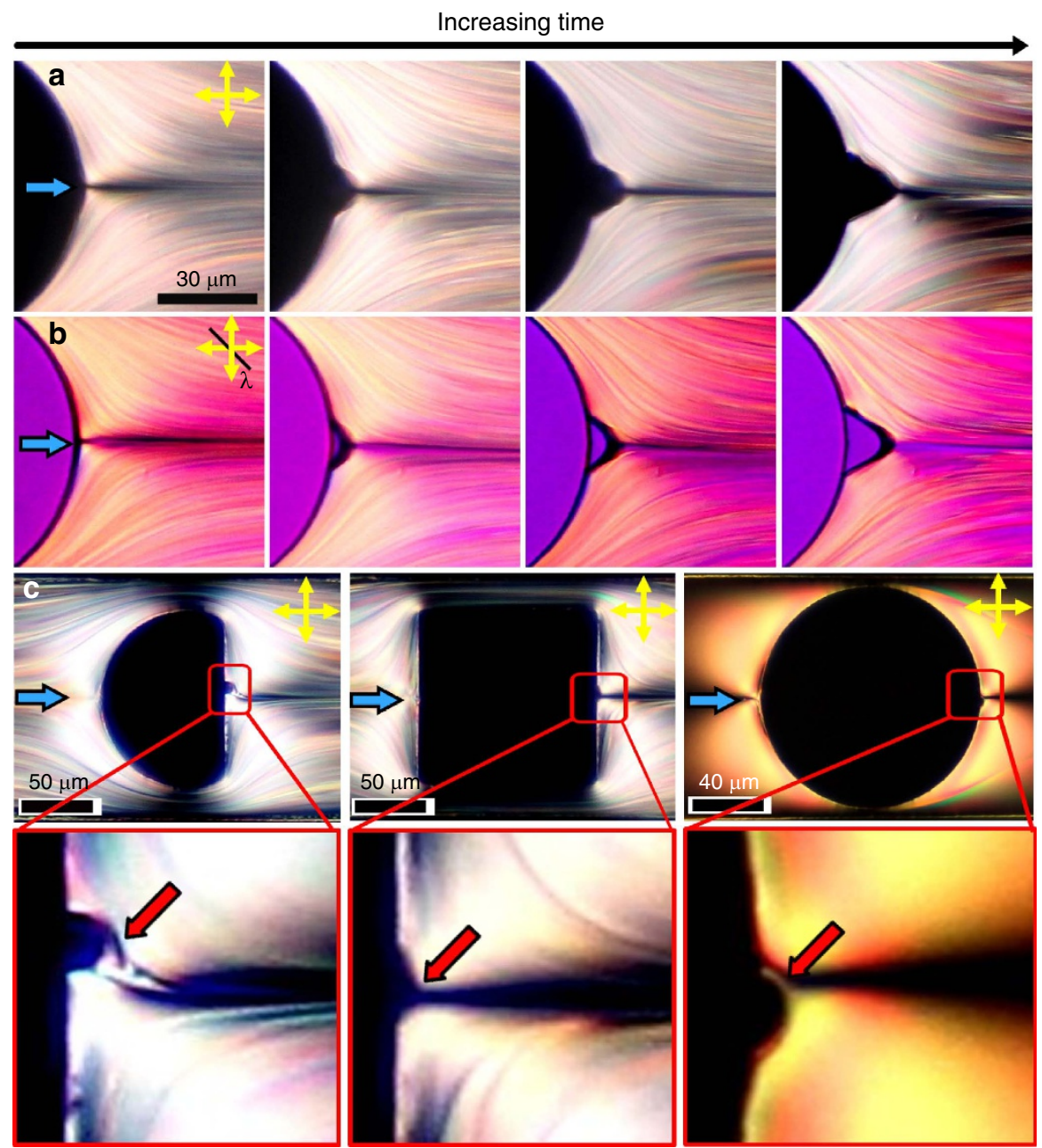

Figure 2 | Cavitation domain growth. (a) Polarized optical micrographs represent the growth of the cavitation volume over time. The total time elapsed is $5 \mathrm{~h}$. The minimum intensity regions (dark appearance) are observed either due to the extinction of the transmitted light as it passes through the vapour phase (in the cavitation domain), or through the bulk nematic phase aligned parallel to one of the polarizers. The two cases are distinguished using a $\lambda$-plate. (b) Introduction of the $\lambda$-plate confirms the absence of the nematic phase in the cavitation domain, and distinguishes it from the bulk nematic aligned parallel to the polarizer. (c) Cavitating domains were observed upon LC flow past different obstacle geometries: semicircle (left) and square (middle); and at different channel depths (right, $d \approx 10 \mu \mathrm{m}$ ). Shallow channels require high Er numbers for cavitation to be triggered.

and, in addition to the roughness of the pillar surface (higher roughness promotes cavitation), it showed a qualitative dependence on the depth of the microchannel. At a given Er, it took longer to reach the saturated volume in a shallow channel $(d<12 \mu \mathrm{m})$, compared with that in deeper channels. However, once formed, the cavitation domain in a shallow channel was observed to be stable for longer times, possibly due to strong pinning of the vapour-liquid interface at the top and bottom surfaces of the microchannel. The POM micrographs with $\lambda$-plate (Fig. $2 b$ ) confirm the absence of the nematic phase within the cavitation domain. While cavitation can be initiated by pre-existing gas bubbles entrapped in the asperities of the confining solid surfaces, we do not expect this to play a determining role in our experiments. Under similar surface conditions, no cavitation was observed when $5 \mathrm{CB}$ in the isotropic phase was flowed, and in experiments with deionized water. The inception of cavitation only in the nematic $5 \mathrm{CB}$ suggests that cavitation is triggered due to the complex anisotropic nature of the flowing material. Cavitation was also reproduced with obstacles having noncircular cross-sections, including semicircular and square geometries (Fig. 2c). At high flow speeds $(\mathrm{Er}>500)$, the domain volume was observed to reduce in size. This is counterintuitive, since high flow speed (high $\mathrm{Re}$ ) is known to favour the onset of cavitation in isotropic fluids. However, in the present setting, we speculate that the strong viscous forces disrupt the pinned vapour-liquid interface, and shear off minuscule gas bubbles from the saturated cavitation domain. This can reduce the domain volume, triggering a dynamic tradeoff between the growth and decay processes that regulate the final cavitation volume at a given Er.

Simulations. To obtain an in-depth understanding of the physical processes that underlie the cavitation phenomenon, we turn to a theoretical modelling of our experimental setup. Due to the limitations in computer performance, the length scales accessible to MD simulations are still too small compared with the micron-sized cavitation domain in our experiments. However, the MD simulations do provide us access to the early stages of the LC cavitation, in addition to the structural information that is not easily tractable with phenomenological theories of LCs 


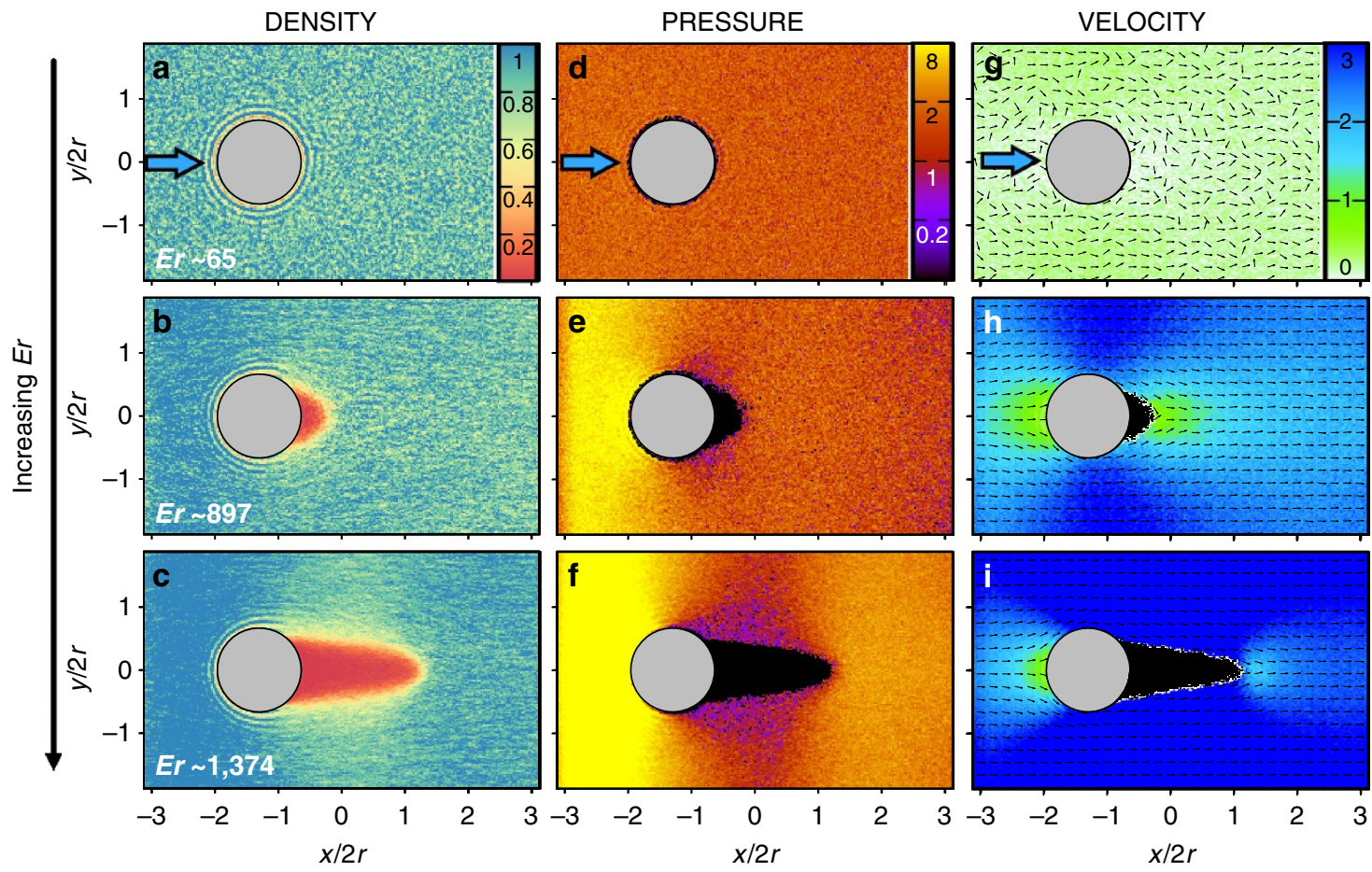

Figure 3 | MD simulation of the hydrodynamic states. (a-c) Maps of the magnitude of local density in the $x-y$ plane located at $z=0$ (see attached color bar). The circle represents the cylindrical pillar and the arrow above each column indicates the direction of flow, at different Ericksen numbers: (a) Er $\simeq 65$, (b) $\mathrm{Er} \simeq 897$ and (c) $\mathrm{Er} \simeq 1,374$. (d-f) MD simulated local pressure plots and (g-i) corresponding local velocity fields, carried out at the same Er as in (a-c). Colour bars attached to plots in the top panel refer to all plots in the same column.

(see Methods and Supplementary Information). To characterize the onset and evolution of cavitation within the LC fluid, in Fig. 3a-c we present the maps of the local density in a $x-y$ cross-section of the system. For the system at rest, that is, without flow, the density is homogeneous throughout the system. For moderate flow conditions the density remains homogeneous across the system (see Fig. 3a). However, at high flow rates, starting at $\mathrm{Er} \simeq 897$, the density across the system starts to change. Figure $3 \mathrm{c}$ shows a well-developed cavitation domain at $\mathrm{Er} \simeq 1,374$. Specifically, we observe a drop in the density in the downstream wake of the pillar (Fig. 3b). The density of this area corresponds to that of vapour phase, and therefore demonstrates hydrodynamic cavitation. The size of the vapour domain first increases till $\mathrm{Er} \simeq 1,374$, and then grows at a reduced rate. At $\mathrm{Er} \simeq 1,586$ the cavitation domain reaches the maximum size and does not grow further.

We notice that the $\mathrm{Er}$ at which cavitation initiates is about a factor of four higher than in our experiments. This apparent discrepancy is resolved by estimating the nematodynamic parameters locally around the micron-sized obstacle, rather than the far-field values. Locally, the Er reaches much higher values due to the increased flow speed through the constriction between the pillar and channel walls ${ }^{44}$. When cavitation first occurs in our experiments, at a far-field Er of 200 $\left(v=800 \mu \mathrm{ms}^{-1}\right)$, the corresponding local Ericksen number $\mathrm{Er}_{\text {loc }}=945\left(v_{\mathrm{loc}}=4,000 \mu \mathrm{m} \mathrm{s}^{-1}\right)$. This value is intriguingly close to $\mathrm{Er}=897$ obtained in the MD simulations. We note that the local Reynolds number still falls within the Stokes regime $\left(\operatorname{Re}_{\mathrm{loc}} \simeq 0.1\right)$. The close agreement between the experiments and simulations prompts us to conclude that the microfluidic experiments and the MD simulations represent equivalent hydrodynamic conditions. Thus, despite the difference in length scales, by analysing the system over comparable nematodynamic conditions, we are able to capture the underlying mechanism for cavitation under relevant boundary conditions.
The development of cavitation depends on the local pressure landscape. Figure $3 \mathrm{~d}-\mathrm{f}$ illustrate maps of the local pressure in the $x-y$ cross-section of the system. The system at rest exhibits a homogeneous local pressure, as one would expect (not shown here). From the map in Fig. $3 \mathrm{~d}$ it is clear that this applies to small Ericksen numbers $\mathrm{Er} \simeq 65$ as well. However, as shown in Fig. $3 \mathrm{e}$, for $\mathrm{Er} \simeq 897$ (the onset of cavitation), this situation changes drastically. On the upstream side, the local pressure builds up because of the flow obstruction by the pillar. This is accompanied by a pressure drop on the downstream side, just behind the cylindrical pillar. After passing the constriction the fluid expands freely, and thus resulting in the drop in local pressure. This effect becomes even more pronounced if flow is further increased as shown in Fig. 3f. Notice that within the cavitation area the local pressure equals to the pressure of an ideal gas.

Figure 4 presents the corresponding evolution of the local pressure in the vertical plane $(y-z$ plane $)$ at specific distances behind the cylindrical pillar. For reasons of symmetry, and to obtain improved statistics, the values of the local pressure have been averaged along the channel depth ( $z$-axis), and are presented across the channel width ( $y$-axis). To avoid confinement effects, we exclude the regions close to the planar substrates from our calculation. For small flow velocities, the local pressure behind the cylindrical pillar is, on average, constant along the $y$-axis. If flow is increased (see Fig. 4a), the local pressure starts decreasing behind the cylindrical pillar. At the threshold value for cavitation, $\mathrm{Er} \simeq 786$, the local pressure attains negative values. Hence, molecules in this area are pushed out. This occurs just before a cavitation volume nucleates. As soon as a cavitation volume is visible $(\mathrm{Er} \simeq 897)$ the local pressure in that area is equal to the ideal gas pressure.

The oscillations visible in Fig. $4 \mathrm{a}$ for $\mathrm{Er} \simeq 65$ are due to the layering effects ${ }^{54,55}$ of the molecules close to the pillar. This leads to changes in pressure in this region located at the centre of the 


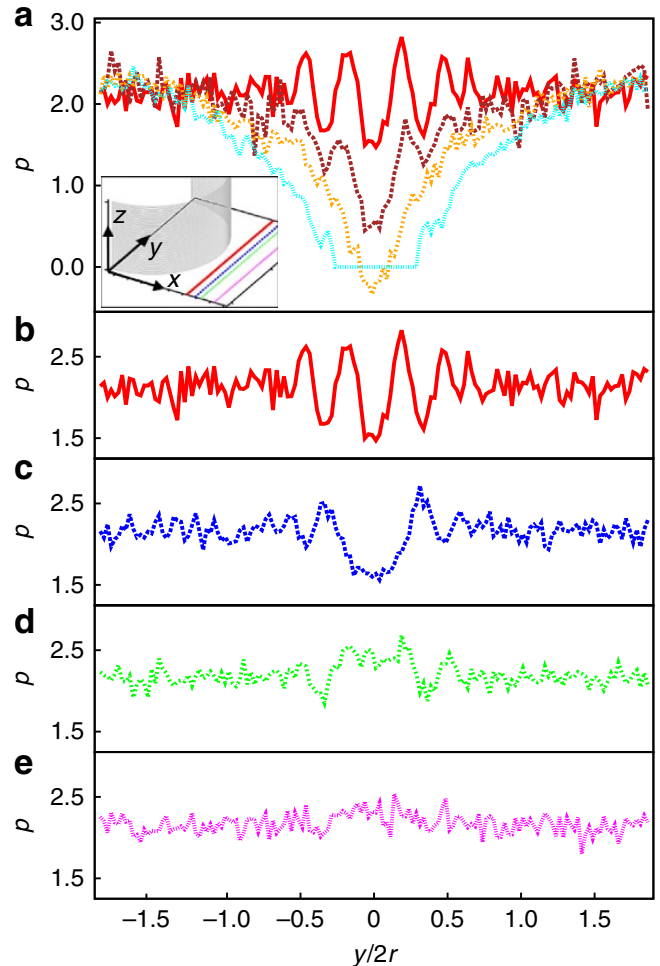

Figure 4 | Mapping the local pressure. (a) Dependence of the local pressure at distance 0.7 behind the surface of the cylindrical pillar in the direction of flow, plotted across the channel width ( $y$-axis) for $E r \simeq 65$ (red line), $\mathrm{Er} \simeq 583$ (brown dashed line) $\mathrm{Er} \simeq 786$ (orange dashed line) and $\mathrm{Er} \simeq 897$ (cyan dotted line). The inset in (a) shows a sketch of different positions behind the cylindrical pillar at which the local pressure was measured. (b-e) Plots of local pressure at specific distances behind the surface of the cylindrical pillar for $E r \simeq 65$ : (b) at a distance of 0.7 behind the pillar, (c) at 1.3, (d) at 1.7 and (e) at 2.7.

$y$-axis. If the local pressure is calculated further away from the surface of the cylindrical pillar, layering effects become less prominent, and as shown in Fig. $4 \mathrm{~b}-\mathrm{e}$, the local pressure minimum (at $y=0$ ) vanishes. From a molecular standpoint, the local pressure oscillations close to the surface of the cylindrical pillar support the nucleation of a cavitation domain. This is consistent with the fact that hydrodynamic cavitation requires a nucleation site such as a surface.

Figure 3g-i maps the local velocity over the mid-plane cross section ( $x-y$ plane) of the nematofluidic system. At small flow rates, $\mathrm{Er} \simeq 65$, the local velocity is rather low, as indicated by the random orientations of the arrows representing the flow direction (Fig. 3g). At the onset of cavitation, $\mathrm{Er} \simeq 897$, a net flow is clearly present (Fig. 3h). The local velocity in the constriction is considerably higher than that in front of and behind the cylindrical pillar. The relative velocity difference becomes stronger at still higher flows. Figure $3 \mathrm{i}$ presents the velocity map at $\mathrm{Er} \simeq 1,374$ in the vicinity of the obstacle and the cavitation domain. Due to lack of sufficient statistics within the gaseous phase, the local convective velocity was set to zero in the cavitation area for calculating the resulting velocity map.

We apply the density maps presented in Fig. 3a-c for estimating the volume, $V_{\mathcal{c}}$, of the cavitation domain. The total volume was obtained by adding up all the cubic volumes for which the local density $\rho_{1} \leq 0.15$. Figure 5 plots the variation of $V_{c}$ with the Reynolds number $R e$, where $\operatorname{Re}_{\mathrm{cr}}$ denotes the critical Reynolds number for the cavitation inception. Alongside anisotropic system described so far, we have also performed

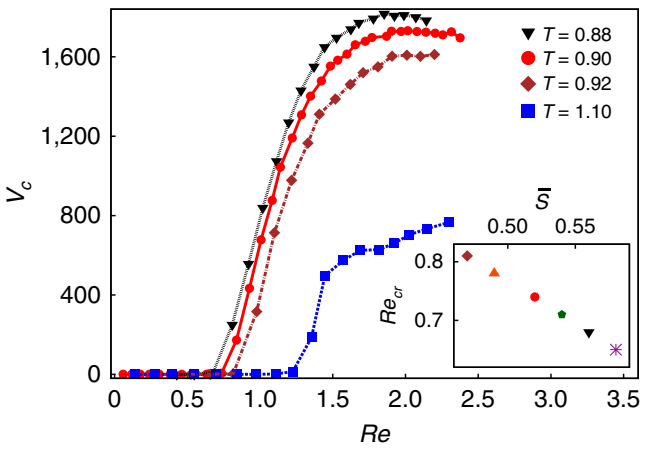

Figure 5 | Growth of cavitation volume. Cavitation volume plotted against Reynolds number Re for the nematic LC at $T=0.88$ (black triangle), at $T=0.90$ (red circle) and at $T=0.92$ (brown diamond), and for an isotropic LC (blue square). The inset shows the critical Reynolds number, $\operatorname{Re}_{c r}$, plotted against the average nematic order parameter $\overline{\mathrm{S}}$, spanning temperatures from $T=0.92$ down to $T=0.87$.

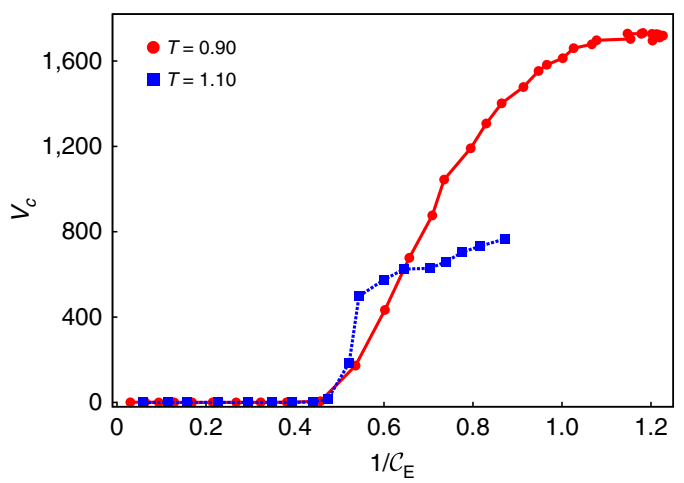

Figure 6 | Cavitation volume and Euler number. Dependence of the cavitation volume on the inverse Euler number $\mathcal{C}_{\mathrm{E}}^{-1}$ for the nematic $\mathrm{LC}$ (red circle) and isotropic LC (blue square).

MD simulations for the same LC model in the isotropic phase $(T=1.1)$. The two systems differ in their viscosity $\eta$ : the nematic LC $(\eta \simeq 37)$ is more viscous than the isotropic LC $(\eta \simeq 21)$. Thus, from the definition of Re, it follows that the nematic LC cavitates at lower critical Reynolds number $\mathrm{Re}_{\mathrm{cr}}$ than the isotropic LC. That fluids with lower viscosity require a larger $\mathrm{Re}_{\mathrm{cr}}$ to cavitate is consistent with our experiments. Neither the deionized water nor $5 \mathrm{CB}$ in isotropic phase elicited cavitation at Reynolds numbers in which the nematic $5 \mathrm{CB}$ cavitated. It is important to note here that, in our simulations, the $\mathrm{Re}_{\mathrm{cr}}$ for the nematic LC is of the same order of magnitude as the local Re in the microfluidic experiments $(\simeq 0.1)$.

For both the nematic and the isotropic phases of liquid crystals, we observe a steep growth of the cavitation domain after its formation, followed by a saturation to a plateau. The height of the saturation plateau is due to the finite size of the simulation box and, therefore, is system size dependent. However, the plateau value of the cavitation domain depends on the nature of the fluid, and can be ascribed to the differences in the liquid matrix structure, for example, different ordering among the three fluids. Figure 5 shows the growth of cavitation domains for the nematic LC at $T=0.88$ and $T=0.92$ that correspond to higher $(\eta \simeq 40)$ and lower $(\eta \simeq 34)$ viscosities, respectively, than what we have considered till now. While the qualitative trend is still retained, quantitative differences emerge, specifically in the growth and saturation of the cavitation domain. The lower the temperature 

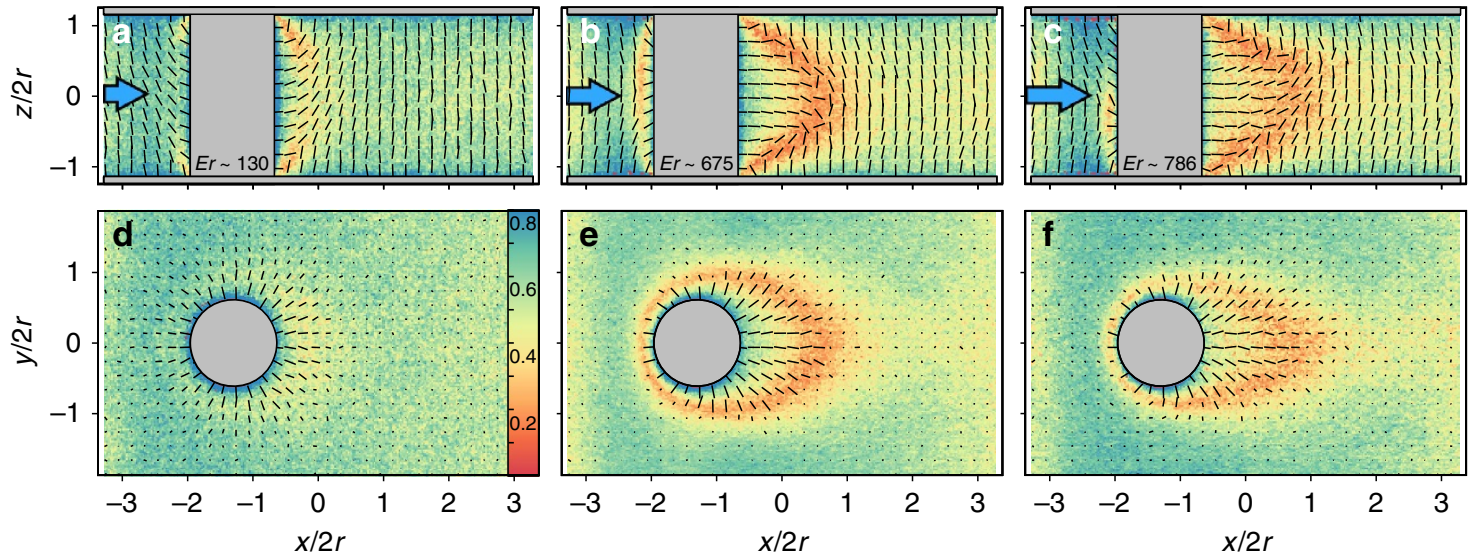

Figure 7 | Flow-induced local nematic order. (a-c) Magnitude of the local nematic order in the $x-z$ plane located at $y=0$ (see attached colour bar). The cylindrical grey area represents the cylindrical pillar and the arrow above each plot indicates the direction of flow. Small dashes in the maps represent the local director field such that the length of a dash denotes its three-dimensional orientation: shorter dashes have an orientation closer to the normal to the plane of the map. The Ericksen numbers considered are (a) $\mathrm{Er} \simeq 130,(\mathbf{b}) \mathrm{Er} \simeq 675$ and (c) $\mathrm{Er} \simeq 786$. (d-f) Corresponding director fields over the $x-y$ plane located at $z=0$.

(higher viscosity), the larger was the resulting cavitation volume. In addition to viscosity, the nematic order parameter also varies with $T$. The inset of Fig. 5 shows the dependence of $\mathrm{Re}_{\mathrm{cr}}$ on the average nematic order parameter $\bar{S}$ for temperatures ranging from $T=0.92$ down to $T=0.87$. For values of $\bar{S}$ in the nematic phase the critical Reynolds number $\operatorname{Re}_{\mathrm{cr}}$ decreases linearly with $\bar{S}$, demonstrating that cavitation is enhanced by stronger nematic alignment. This qualitative measurement serves as a first step towards understanding the influence of long range ordering on cavitation at micro scales.

Figure 6 shows the cavitation volume $V_{\mathrm{c}}$ in terms of the inverse Euler number $\mathcal{C}_{\mathrm{E}}$, a dimensionless number that is independent of the viscosity of the system. The ratio between the pressure gradient and the kinetic energy per volume is decisive for the development of a cavitating volume, as captured distinctly in Fig. 6. Independent of the model system cavitation occurs at the same Euler number $\mathcal{C}_{\mathrm{E}}$. When cavitation first occurs in the experiments we find $\mathcal{C}_{\mathrm{E}} \simeq 0.5$ that is within similar order of magnitude as the simulations.

Figure 7 maps the local nematic order parameter over the range of flow regimes considered here. The flow field reorients the nematic director from homeotropic (on the surfaces) to flow-aligned orientation (in the flowing matrix). Topological defects arise in the director field, close to the top and the bottom walls, where the cylindrical pillar intersects the channel surfaces (Fig. 7a). The singular defect loops are formed due to the homeotropic anchoring, both on the channel surfaces and on the pillar, and are consistent with the defect topology discussed in ref. 49. It is evident from Fig. $7 \mathrm{~d}$ that over the $x-y$ plane, located at the channel half depth $(z=0)$, no defect is visible. The director field remains stable for small Er. However, for $\mathrm{Er} \geq 675$ a single loop around the pillar stabilizes in the $x-y$ mid-plane. The loop is deformed and extended towards the downstream direction along with the flow, shown in Fig. 7b,e. Additionally, there is a growing region of flow alignment behind the cylindrical pillar in the downstream direction. Upon increasing Er one can see that the loop becomes stretched further downstream (see Fig. 7c). However, the overall defect topology, especially downstream behind the pillar, is increasingly smeared, possibly due to the appearance of the vapour phase at high Ericksen numbers (Fig. 7f). Changing the surface anchoring from homeotropic to planar did not produce any qualitative change in our results. This agrees well with the experiments, where too we have observed that cavitation in nematic phase was independent of the nature of the surface anchoring.

Theoretical analysis. We theoretically study the cavitation in an anisotropic matrix and predict the role of nematic order parameter on cavitation inception. As the simplest case, let us consider a spherical environment containing the nematic liquid crystal (see Methods and Supplementary Fig. 1). The fluid is subject to a pressure drop $\Delta P$. Under appropriate hydrodynamic conditions, a stationary pressure drop leads to cavitation in the nematic bulk. The first region of interest, the spherical cavitation domain with radius $r_{\mathrm{c}}$, encloses the vapour phase of the LC. From our foregoing experiments and computer simulations we know that the nematic LC molecules align homeotropically to the surface of the vapour phase. This we consider as the region 2: a spherical shell of thickness $r_{\mathrm{d}}-r_{\mathrm{c}}$ with perfect homeotropic alignment to region 1. Finally, the rest of the system is the bulk nematic phase that we will treat in the mean-field approximation, namely, with a homogeneous nematic order parameter S. Locally, the order parameter may vary as a result of the inception and growth of the cavitation domain. The third region has a radius $r_{\mathrm{L}}>r_{\mathrm{d}}$, and excludes the sphere of radius $r_{\mathrm{d}}$.

Although the presence of hydrodynamic flow induces nonequilibrium conditions, the fluid has typically enough time to reach conditions of local equilibrium, and therefore a discussion based on free energies is admissible. We find that the total free energy can be expressed as

$$
\begin{aligned}
F_{\text {tot }} & =\frac{1}{2} \alpha\left[S_{\mathrm{N}}^{2} v^{1 / 3}+\left(8 \pi \frac{\tau}{\alpha} r_{\mathrm{L}}^{2}+\frac{\beta}{\alpha}-\frac{\alpha S_{\mathrm{N}}^{2}}{\gamma}\right) v^{2 / 3}\right. \\
& \left.+\left(-\frac{8}{3} \pi r_{\mathrm{L}}^{3} \frac{\Delta P}{\alpha}+\frac{\alpha^{2} S_{\mathrm{N}}^{2}}{\gamma^{2}}\right) v\right]
\end{aligned}
$$

where the cavitation volume $v \equiv \delta^{3} \equiv\left(r_{\mathrm{c}} / r_{\mathrm{L}}\right)^{3}, \alpha, \beta$ and $\gamma$ are coefficients with dimensions of energy (see Methods section for details), $\tau$ the surface tension of the liquid-vapour interface, $S_{\mathrm{N}}$ is the nematic order parameter in the absence of cavitation and $\Delta P$ is the absolute value of the pressure drop. Cavitation will occur when the free energy in equation (1) develops a minimum $v^{*}>0$. This critical point is found from the two conditions $\partial F_{\text {tot }} / \partial v=0$ and $\partial^{2} F_{\text {tot }} / \partial v^{2}=0$ that determine the 
critical cavity volume and critical pressure

$$
v^{*}=-\left(\frac{\lambda}{\mu}\right)^{3}, \quad \frac{\Delta P^{*}}{P_{0}}=\frac{\alpha^{2}}{\gamma^{2}} S_{\mathrm{N}}^{2}-\frac{1}{3} \frac{\mu^{2}}{\lambda},
$$

where $P_{0} \equiv \alpha /\left(\frac{8}{3} \pi r_{\mathrm{L}}^{3}\right)$ is a reference pressure, $\lambda \equiv \frac{1}{2} \alpha S_{\mathrm{N}}^{2}$, $\mu \equiv \frac{1}{2} \alpha\left(8 \pi \frac{\tau}{\alpha} r_{\mathrm{L}}^{2}+\frac{\beta}{\alpha}-\frac{\alpha S_{\mathrm{N}}^{2}}{\gamma}\right)$. For $\lambda>0$ and $\mu<0$ a finite minimum develops.

The influence of the order parameter on cavitation can be found from studying how $v^{*}$ depends on $S_{\mathrm{N}}$,

$$
\begin{aligned}
v^{*} & =-\left(\frac{\lambda}{\mu}\right)^{3}=-\left[\frac{S_{\mathrm{N}}^{2}}{8 \pi \frac{\tau}{\alpha} r_{\mathrm{L}}^{2}+\frac{\beta}{\alpha}-\frac{\alpha S_{\mathrm{N}}^{2}}{\gamma}}\right]^{3} \\
& \simeq\left(\frac{\gamma}{\alpha}\right)^{3}\left(1+3 \frac{c \gamma}{\alpha S_{\mathrm{N}}^{2}}\right),
\end{aligned}
$$

where $c \equiv 8 \pi \frac{\tau}{\alpha} r_{\mathrm{L}}^{2}+\frac{\beta}{\alpha} \ll 1$. Thus, as $S_{\mathrm{N}}$ grows, $v^{*}$ decreases and cavitation grows easier. This fact is confirmed by our computer simulations.

The following physical picture emerges from the theory above. Upon increasing flow speeds the pressure in the stagnation point downstream of the obstacle diminishes. The elastic energy associated with the nematic order increases due to the deformations induced by both the flow alignment and the pressure drop. At a certain flow magnitude, it becomes energetically more favourable to produce a liquid-gas interface whereon anchoring conditions compatible with the nematic director alignment can materialize. Consequently, the director field can relax, and the nematic order parameter attains values locally higher and closer to the value in stationary conditions than in the case when no liquid-gas interface would form. We note that the presence of the coupling $\alpha$ between order parameter and deformation is crucial to produce nematic cavitation. At vanishing $\alpha$, there is no advantage in producing cavitation. Note also that for vanishing $S_{\mathrm{N}}$ (that is, not in the nematic phase) cavitation does not occur. Our theoretical arguments are in fact valid only for phases with nematic order.

\section{Discussion}

We report hydrodynamic cavitation in the Stokes flow regime of an anisotropic fluid, due to flow of a nematic LC past a micronsized pillar within a microfluidic chip. By varying the flow speed within a channel, we studied the growth of a stable, microscopic cavitating domain on the pillar surface that positioned stably at the downstream stagnation point of the micropillar. Cavitating domains were experimentally observed for $200<\operatorname{Er}<500$, and $\operatorname{Re} \ll 1$, in microchannels possessing both planar and homeotropic boundary conditions. For flows with $\mathrm{Er}<200$, cavitation was not detected, whereas for $\mathrm{Er}>500$, the cavitating domain, after formation, was sheared off due to the high flow speed, and advected downstream. At a given $\mathrm{Er}$, the cavitation domain, over a few hours $(5 \mathrm{~h}$ for $\mathrm{Er}=250)$, attained the saturation volume. By demonstrating that cavitation in complex anisotropic fluids can be triggered at $\operatorname{Re} \simeq 10^{-3}$-a hydrodynamic Stokes flow regime, our work complements earlier reports on hydrodynamic cavitation of isotropic fluids at high Reynolds numbers (for example, microchannel flows in deionized water flowing through a micro-orifice by Mishra and Peles ${ }^{56-58}$ ). The occurrence of cavitation under both planar and homeotropic boundary conditions indicates that the nematofluidic conditions that favour cavitation in $5 \mathrm{CB}$ are weakly influenced by the nature of LC anchoring on the microchannel surfaces. This corroborates that the viscous forces play a dominant role relative to the elastic forces, given the high Er at which inception of cavitation is observed in our experiments.

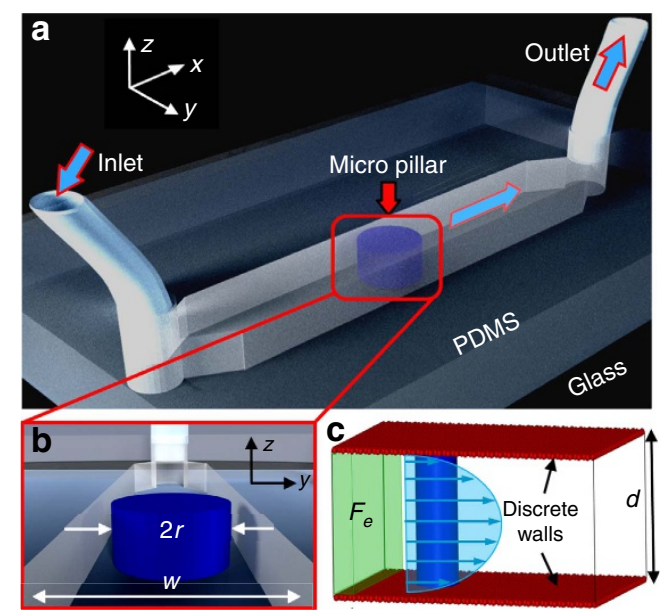

Figure 8 | Microfluidic confinement. (a) Microchannel with micron-sized cylindrical pillar fabricated using surface bonding of PDMS and glass components. The orthographic projection shows a linear channel of a rectangular cross-section with the integrated micropillar. The $x, y$ and $z$ coordinates denote the flow direction of the anisotropic fluid (a nematic molecule), the transverse direction of the channel (width) and the channel depth, respectively. The blue arrowheads show the flow path.

(b) Microchannel projection showing the cross-sectional view (yz plane) with $r, w$ and $d$ being the pillar radius, channel width and depth, respectively. (c) Sketch of the empty simulation channel with the discrete walls.

Using MD simulations, we have established a critical Reynolds number criterion, $\mathrm{Re}_{\mathrm{cr}}$, that sets the threshold $\mathrm{Re}$ for cavitation inception. Strikingly, the simulations reveal that the $\operatorname{Re}_{\mathrm{cr}}$ can be up to $50 \%$ lower in anisotropic fluids than in isotropic fluids. While the susceptibility of a liquid to cavitate is clearly linked to its viscosity, importantly still, our finding that $\operatorname{Re}_{\mathrm{cr}}$ decreases linearly with the global nematic order parameter $\bar{S}$ demonstrates that long-range ordering is a key material parameter for cavitation inception in complex anisotropic fluids. Measurements of the growth in cavitation volume revealed that the qualitative behaviour in isotropic LC fluids is similar to the one of anisotropic fluids, for example, the rate of volume growth. However, the size of cavitation domain at large Er numbers is markedly different. This further adds on to the consideration that structural differences of the system, such as long-range order, affect the growth of cavities due to hydrodynamic flow. In addition, our simulations at lower Er numbers (before cavitation sets in) are in very good agreement with earlier microfluidic experiments ${ }^{49}$. Finally, our theoretical calculations support the occurrence of cavitation in nematic LCs, and correctly predict the lowering of the cavitation threshold with increasing nematic order parameter.

Our study is a step towards understanding cavitation in anisotropic fluids that aptly complements investigations on cavitation in isotropic fluids. Beyond its fundamental significance, this study can potentially introduce a novel control parameter for devising applications based on cavitation at low Reynolds number flows and in the development of future biomedical applications.

\section{Methods}

Materials. We have used 4'-pentyl-4-biphenylcarbo-nitrile, commonly known as 5CB (Synthon Chemicals), as the anisotropic liquid for our experiments. 5CB is a single component LC that is in the nematic phase at room temperature, and was used without any additional purification. In the nematic phase, the $5 \mathrm{CB}$ molecules possess a long-range orientational order that is lost at higher temperatures, as 5CB undergoes a transition to the isotropic phase at $\sim 33^{\circ} \mathrm{C}$ (ref. 59). On cooling it down below $18^{\circ} \mathrm{C}$, nematic $5 \mathrm{CB}$ undergoes a phase transition to the crystalline phase. 
Microfluidic setup. The microfluidic devices were fabricated using channel reliefs cast on polydimethylsiloxane (PDMS, Sylgard 184, Dow Corning) and surface bonded to glass following exposure to oxygen plasma. The cylindrical pillar was formed as a part of PDMS cast prepared using soft lithography ${ }^{49}$. An orthographic view of the microfluidic chip is shown in Fig. 8a. Experiments were conducted in simple linear channels with a rectangular cross-section and a cylindrical pillar placed at the middle of the channel, spanning the channel depth, $d$, from 10 to $50 \mu \mathrm{m}$, and the pillar diameter, $2 r$, between 10 and $80 \mu \mathrm{m}$ (see Fig. $8 \mathrm{~b}$ ). Here we present results that were obtained (unless otherwise stated) due to the flow of nematic 5CB past micropillars of diameter, $2 r=80 \mu \mathrm{m}$ within $w \approx 100 \mu \mathrm{m}$ wide, $d \approx 15 \mu \mathrm{m}$ deep, and sufficiently long $(20 \mathrm{~mm})$ channels. In Fig. $8 \mathrm{a}, x, y$ and $z$ denote the flow direction, channel width and channel depth, respectively. Cylindrical holes of $750 \mu \mathrm{m}$ diameter at either ends of the channel served as the housing for the inlet and outlet tubes (Teflon). The tubes $(300 \mu \mathrm{m}$ inner diameter and $760 \mu \mathrm{m}$ outer diameter) were inserted within the holes punched in the PDMS mould. Smaller diameter of the PDMS housing as compared with the external diameter of the tube ensured good mechanical fitting. Additionally, uncured PDMS was applied at the point of fitting. After curing of the PDMS, any possible leakage at the inlet and outlet ports was ruled out. For filling the channel and subsequent flow experiments, a gear pump (neMESYS, Cetoni) with flow rate precision of $\mathrm{nlh}^{-1}$ ( $\pm 0.02 \%$ of the corresponding flow rates) was used to drive a gas-tight microlitre syringe (1001LT, Hamilton Bonaduz), connected to the inlet tube. The outlet tubing was dipped in a sink of 5CB. In agreement with the observed dependence of the swelling ratio on the substance polarity $(\simeq 4.8 \mathrm{D} \text { for } 5 \mathrm{CB})^{60}$, no perceptible swelling of PDMS by $5 \mathrm{CB}$ was observed during the course of our experiments. To rule out the presence of pre-existing gas or air pockets entrapped in the surface asperities, and anywhere else in the fluidic circuit, the experiments were conducted after degassing the PDMS-glass microchannels, connected to the LC-filled microfluidic syringe, for $1 \mathrm{~h}$ using a vacuum chamber. In addition to visualizing the cylindrical pillar, the entire microfluidic device was periodically scanned to check for other cavitation-like nucleation sites.

Surface anchoring and flow field characterization. The microfluidic devices employed here comprised two chemically distinct surfaces, namely PDMS and glass. While LC molecules orient perpendicular to the PDMS surface (homeotropic surface anchoring), on the glass surface, the molecules anchor parallel to the glass surface with azimuthal degeneracy (degenerate planar anchoring) ${ }^{48}$. To achieve a homogeneous homeotropic surface anchoring on all the surfaces, the confinement was functionalized using an aqueous solution of octadecyldimethyl(3-

trimethoxysilylpropyl)ammonium chloride (DMOAP). The channel was first filled with the DMOAP solution and then rinsed with deionized water (after $\approx 10 \mathrm{~min}$ ). The surface treatment was thereafter thermally stabilized by heating the channel at $80^{\circ} \mathrm{C}$ for $15 \mathrm{~min}$ and at $110^{\circ} \mathrm{C}$ for $1 \mathrm{~h}$. This yielded homogeneous homeotropic surface anchoring conditions on all the surfaces. To induce planar boundary conditions, freshly fabricated microchannels were rinsed with a dilute solution of polyvinyl alcohol (average molecular weight $=90 \mathrm{k}$, concentration $0.1 \%$ by weight in deionized water). The hydroxyl groups of polyvinyl alcohol bond covalently to silanol groups on the glass surface and plasma-treated PDMS surfaces ${ }^{59}$. The bonding was additionally stabilized by baking the filled channel at $80^{\circ} \mathrm{C}$ for $15 \mathrm{~min}$ followed by baking at $110^{\circ} \mathrm{C}$ for $1 \mathrm{~h}$. On completion of the baking process, the polymer chains, oriented parallel to the confining surfaces, imposed a planar surface anchoring on all four walls of the microchannel.

We have combined white light microscopy in bright-field mode and POM to identify the cavitating domain and the director orientation of the flowing LC molecules, using a Nikon Eclipse LV100 polarizing microscope. Imaging was carried out with a $20 \times$ air objective, $\mathrm{NA}=0.45$. The flow speed was typically extracted from high-speed videos of flowing particles using standard particle tracking techniques ${ }^{59}$. Control experiments, to check for long-term effects of 5 CB flow on PDMS surfaces, were also done to specifically identify any surface degradation of PDMS due to $5 \mathrm{CB}$ (which can potentially act as a nucleation site for cavitation). We report no surface degradation of PDMS due to flowing $5 \mathrm{CB}$, for at least $24 \mathrm{~h}$ of continuous flow.

Light travelling through a singular disclination in liquid crystal can lead to lensing effects that renders the defect optically distinct from the neighbouring ordered region ${ }^{61}$. In high Er nematic flows, disclinations give rise to numerous flowing optical lenses within the flow-aligned nematic matrix. We visualize such disclination lines using video microscopy, close to the micron-scale obstacle. By means of image analysis technique we capture the differential intensity between the frames acquired from the video, and average them over the duration of the video acquisition. Thereby, we obtain a time-averaged differential intensity map of the disclination lines flowing past the obstacle, as shown in Fig. 1c. This information was used as a proxy for the streamlines of the nematic flow, since disclinations-both singular and escaped structures-are stretched due to the viscous drag and, in general, appear as straight lines within the flow ${ }^{62}$

\section{Material and nematofluidic parameters. Microfluidic devices, possessing} homeotropic and planar surface anchoring, were initially filled with $5 \mathrm{CB}$ in the isotropic state, and allowed to equilibrate to nematic phase at room temperature. Thereafter, we progressively increased the volume flow rate $Q\left(0.01 \mu \mathrm{h} \mathrm{h}^{-1}\right.$ $<Q<20 \mu \mathrm{l} \mathrm{h}^{-1}$ ) to detect the first appearance of the microscopic cavitating domain. For a typical channel with dimensions, $w=100 \mu \mathrm{m}$, and $d=15 \mu \mathrm{m}$, the corresponding flow speed, $v$, within the microchannel varied between $\simeq 2 \mu \mathrm{m} \mathrm{s}^{-}$ and $4 \mathrm{~mm} \mathrm{~s}^{-1}$. Thus, for the nematic LC 5CB having bulk dynamic viscosity, $\eta \simeq 50 \mathrm{mPa} \mathrm{s}{ }^{59}$, the characteristic Reynolds number $\mathrm{Re}=\rho v l / \eta$ ranged between $10^{-6}$ and $10^{-3}$ that, from a hydrodynamic point of view, signifies the Stokes flow regime. Here, $\rho \simeq 1,025 \mathrm{~kg} \mathrm{~m}^{-3}$ is the material density, and $l=4 w d / 2(w+d) \approx 26 \mu \mathrm{m}$ is the hydraulic diameter of the rectangular microchannel. The Ericksen number, a dimensionless number that captures the relative importance of the viscous effects over elastic effects in LC flows ${ }^{44}$, is given by $\mathrm{Er}=\eta v l / K$, where $K=5.5 \mathrm{pN}$ is the single elastic constant of nematic $5 \mathrm{CB}$. It is important to note that the elastic constant does not appreciably change at the constrictions of the microfluidic channel. Elastic constants depend on the nematic order parameter, and there should be no other dependence unless the short-range, molecular structure of the fluid is perturbed. For flow-aligning liquid crystals, the balance of hydrodynamic torque with the elastic one selects a steady-state angle. In this way, the viscous stresses relax to a local equilibrium that does not alter the microphysics of the fluid. We do not expect that the hydrodynamic flow in our experiments, although strong, change the short-range structure of the fluid. From the field map of the nematic order parameter calculated via molecular dynamics simulations and shown in Fig. 7, we can observe that in fact the local order parameter at the constrictions exhibits no particular difference from the values sufficiently away from the micropillar. We can then conclude that the elastic constants will not significantly change in the constrictions.

In our experiments, the corresponding values of Er ranged from $\simeq 0.5$ to 850 : for $\mathrm{Er}<1$, the elastic effects outweighed the viscous effects, whereas at $\mathrm{Er}>1$, the viscous effects are dominant over their elastic counterpart. As Re and Er are both proportional to $v l$, we immediately find that $\mathrm{Re}=K \rho / \eta^{2}$ Er. Inserting the typical numbers for the materials properties of LCs, we find a general conversion factor: $\mathrm{Re} \simeq 10^{-4} \mathrm{Er}$, for $K \simeq 10^{-11}$, obtained as a ratio of interaction energy between molecules and the molecular distance, $\rho \simeq 10^{3}$ and $\eta \simeq 10^{-2}$ (ref. 44). Therefore, the specific conversion factor depends on the material properties of the particular LC and can vary for different LC compounds. For $5 \mathrm{CB}, \mathrm{Re} \simeq 10^{-6} \mathrm{Er}$. Nevertheless, the experiments and simulations remain in the laminar flow regime for all Er considered.

MD simulations. We consider a confined fluid with a cylindrical pillar filled with a fluid composed of $N$ molecules (see Fig. 8c). The system is confined by atomically resolved solid substrates that induce friction on the flowing molecules to obtain a steady state during the course of a simulation.

In this work all quantities of interest are given in the usual dimensionless (that is, 'reduced') units, by taking the molecule's mass $m$, diameter $\sigma$ and potential energy scale $\varepsilon$ as the basic units of mass, length and energy, respectively. All simulations reported here have been carried out for a LC fluid containing $N=2.4 \times 10^{4}$ molecules in a volume $V=s_{x} s_{y} s_{z}$. We choose a Cartesian reference system oriented as in the experiments. To ensure that the LC fluid is sufficiently deep in the nematic phase we choose a temperature $T=0.90$ and, under isothermal-isobaric conditions, a pressure $P=1.80$. For this thermodynamic state point we obtain a mean number density of $\bar{\rho}=N /\langle V\rangle \simeq 0.8$ in the bulk.

MD simulations generate molecular configurations by integrating Newton's equations of motion for each particle in the fluid subject to the total potential energy due to other particles, confining substrates and external potentials. The differential equations are solved through a velocity-Verlet algorithm with a time step of $\delta t=10^{-3}$ specialized for $\mathrm{LCs}^{63}$. A nonequilibrium, steady-state Poiseuille flow is induced along the $x$-axis by applying a body force $\mathrm{F}_{\mathrm{e}}=F_{\mathrm{e}} \hat{\mathbf{e}}_{x}$ in a thin slice of thickness $\delta s_{x}=2.00$ located at $x=-s_{x} / 2+\delta s_{x}$ at one end of the simulation cell and upstream of the cylindrical pillar (see Fig. $8 c$ ).

Every simulation is performed according to the following protocol: first, we equilibrate the LC fluid in the isothermal-isobaric (NPT) ensemble without flow second, a hydrodynamic flow is generated via a pressure gradient and a second equilibration run is performed in the canonical (NVT) ensemble to reach the steady state; finally, we perform the production run in the NVT ensemble, and calculate all physical observables discussed below. For the computation of local quantities we divide our system by means of a grid with cubic cells of side length 0.2 .

Because of the external body force, the system needs to be thermostatted permanently to achieve a stationary nonequilibrium state. As explained in detail in previous studies ${ }^{64,65}$, the choice of a thermostat capable of efficiently removing viscous heating is of vital importance here because of the presence of the cylindrical pillar. Following our previous work we employ the Galilean-invariant thermostat proposed by Stoyanov and Groot ${ }^{66}$ that, because of its Galilean-invariant nature, conserves momentum locally.

The channel aspect ratio and relative dimension of the microfluidic components were maintained in the simulation domain for MD simulations. Figure $8 \mathrm{c}$ shows the sketch of the empty simulation channel with a cylindrical pillar. The channel is confined by discrete walls that are atomically resolved solid substrates that induce friction on the flowing molecules. The green shaded volume marks the region where a body force $F_{\mathrm{e}}$ is applied to create the Poiseuille flow. We have carried out two sets of simulations: first, where the walls possess homeotropic surface anchoring conditions and, second, where the walls possess planar surface anchoring conditions. 
Static and dynamic observables. We first consider the pressure tensor

$$
\begin{aligned}
\mathbf{P}(\mathbf{r}) & =\frac{m}{V}\left\langle\left[\sum _ { i = 1 } ^ { N - 1 } \left(\left(\mathbf{v}_{i}-\mathbf{v}\right) \otimes\left(\mathbf{v}_{i}-\mathbf{v}\right) .\right.\right.\right. \\
& \left.\left.\left.+\sum_{j=i+1}^{N} \mathbf{r}_{i j} \otimes \mathbf{F}_{i j}\right)\right] \delta\left(\mathbf{r}_{i}-\mathbf{r}\right)\right\rangle
\end{aligned}
$$

where $\mathbf{v}_{i}$ is the molecule's velocity vector, $\mathbf{v}=v \hat{\mathbf{e}}_{x}$ is the averaged local flow velocity, $\delta$ is the Dirac $\delta$-function, $\otimes$ the tensor product, $\mathbf{r}_{i j}=\mathbf{r}_{i}-\mathbf{r}_{j}$ the distance vector between the centres of mass of molecules $i$ and $j$, respectively, $\mathbf{F}_{i j}$ describes the force acting between those molecules, and $\langle\ldots\rangle$ indicates a time average. Notice the convective part of the velocity is subtracted from the kinetic energy contribution in equation (4) and one can therefore apply standard concepts of equilibrium thermodynamics. The scalar pressure is defined by $P \equiv \frac{1}{3} \operatorname{Tr} \mathbf{P}$. For a gas the contribution of the internal forces in equation (4) becomes negligible. Therefore, the pressure within the cavity is equivalent to the corresponding ideal gas pressure, $P=N k_{\mathrm{B}} T / V$.

To analyse the deformation of the director field caused by the presence of the cylindrical pillar and the associated local nematic order we adopt the local alignment tensor defined as ${ }^{67}$

$$
\mathbf{Q}(\mathbf{r})=\frac{1}{2 \rho(\mathbf{r})}\left\langle\sum_{i=1}^{N}\left[3 \hat{\mathbf{u}}_{i} \otimes \hat{\mathbf{u}}_{i}-\mathbf{1}\right] \delta\left(\mathbf{r}_{i}-\mathbf{r}\right)\right\rangle,
$$

which is a real, symmetric and traceless second-rank tensor. $\mathbf{Q}(\mathbf{r})$ can be represented by a $3 \times 3$ matrix, where $\hat{\mathbf{u}}_{i}$ is a versor (unit vector) specifying the orientation of molecule $i$ in a space-fixed frame of reference. In equation (5) $\rho(\mathbf{r})$ is the local (number) density. The local alignment tensor satisfies an eigenvalue equation that we solve analytically. With these solutions we define the local nematic order parameter $S(\mathbf{r})$ as the largest eigenvalue and the associated eigenvector as the local nematic director $\hat{\mathbf{n}}(r)$.

As in the experiments we characterize the state of the LC fluid under nonequilibrium steady-state conditions with the Reynolds number $\operatorname{Re}=\bar{\rho} v l / \eta$, and the Ericksen number $\mathrm{Er}=\eta \nu l / K$, where we take $l$ as the distance between the planar solid substrates. To determine the shear viscosity $\eta$ and the elastic constant $K$ of our simulated LC we proceed as in our earlier study ${ }^{64}$.

Finally, to characterize the potential of the LC fluid to cavitate we use the so-called Euler number ${ }^{68} \mathcal{C}_{\mathrm{E}}=\frac{\Delta P}{\bar{\rho} v^{2}}$ where $\Delta P$ is the pressure difference between the upstream and downstream end of the simulation box. The Euler number quantifies the ratio of forces due to the local pressure gradient and the kinetic energy per volume.

Free energy. The total free energy of the system can be written as

$$
F_{\text {tot }}=F_{1}+F_{2}+F_{3},
$$

where the indices 1,2 and 3 refer to the three domains in which the system is divided according to the discussion in the Results section (see Supplementary Fig. 1). The free energy associated to the cavitation domain, region 1 , is

$$
F_{1}=-\frac{4}{3} \pi r_{\mathrm{c}}^{3} \Delta P+4 \pi r_{\mathrm{c}}^{2} \tau,
$$

where $\Delta P$ is the absolute value of the pressure drop and $\tau$ the surface tension of the liquid-vapour interface. In the following it will be useful to introduce a scaled measure of the cavitation domain $\delta \equiv r_{\mathrm{c}} / r_{\mathrm{L}}$. We can then rewrite $F_{1}$ as

$$
F_{1}=-\frac{4}{3} \pi \Delta P r_{\mathrm{L}}^{3} \delta^{3}+4 \pi \tau r_{\mathrm{L}}^{2} \delta^{2} .
$$

In region 2, the liquid crystal is homeotropically aligned to the surface of the vapour phase. We consider here a perfectly aligned spherical shell of thickness $r_{\mathrm{d}}-r_{\mathrm{c}} \equiv \epsilon=$ const $\ll r_{\mathrm{c}}$. The free energy associated to region 2 stems simply from the Frank free energy (in the one constant $K$ approximation)

$$
F_{2}=\frac{1}{2} K \int\left[(\nabla \cdot \mathbf{n})^{2}+(\nabla \times \mathbf{n})^{2}\right] \mathrm{d} V,
$$

where $\mathbf{n}$ is the versor representing the director. After integrating, $F_{2}$ turns out to be a constant, $8 K\left(r_{\mathrm{d}}-r_{\mathrm{c}}\right)$, so that it can be ignored in the following.

In region 3 the fluid is modelled as a homogeneous nematic liquid crystal with a constant value of the nematic order parameter. Because of the presence of the cavitation, however, the orientational field of the fluid is deformed. This is due to the fact that the boundary conditions for the nematic alignment are fixed. At the fluid-vapour interface they must be homeotropic, whereas in the bulk of region 3 a homogeneous nematic fluid with a fixed global director is assumed. We consider a mean-field approximation for the treatment of the fluid for $r>r_{\mathrm{d}}$ so that the fluid is characterized simply by the value of its nematic order parameter $S$. This is obviously a simplifying approximation. The stresses induced by cavitation can be relaxed by reorientations of the local director field. We ignore these effects here to keep the theoretical treatment tractable and bring to the front the relevant physics.

With these provisos, we can now build the free energy for region 3 . First, there must be a coupling term between the deformation introduced by the cavitation, $\delta$, and the global nematic order parameter $S$. At the lowest order in $\delta$ this must be of the form $\frac{1}{2} \alpha S^{2} \delta$. This term reflects the fact that $S$ decreases as the relative size of the cavitation increases because the liquid crystal must accommodate two conflicting boundary conditions in an increasingly small volume. The fluid must additionally exert a 'resistance' to being deformed by the cavitation because of its nematic nature. We model this effect simply with a term $\propto \delta^{2}$. The last contribution comes from the Landau-de Gennes free energy of a nematic fluid $F_{\mathrm{LdG}}=\frac{A}{2} S^{2}-\frac{B}{3} S^{3}+\frac{C}{4} S^{4}$, where $A, B$ and $C>0$.

The 'deformation' free energy for region 3 can then be written as

$$
F_{\text {def }}=\frac{1}{2} \alpha S^{2} \delta+\frac{1}{2} \beta \delta^{2}+\frac{1}{2} \gamma\left(S-S_{\mathrm{N}}\right)^{2} .
$$

Because the fluid is maintained in the nematic phase, in the last term on the right-hand side of equation (10) we replace $F_{\mathrm{LdG}}$ with the minimum associated to the nematic phase that is found from the condition $\partial F_{\mathrm{LdG}} / \partial S=0$, and where the order parameter of the nematic phase $S_{\mathrm{N}} \equiv \frac{1}{2 C}\left(B+\sqrt{B^{2}-4 C A}\right)$ and $\gamma=-2 A+\frac{B}{2 C}\left(B+\sqrt{B^{2}-4 C A}\right) . S$ is determined by the deformation $\delta$, that is, it is found by minimizing $F_{\text {def }}, \partial F_{\text {def }} / \partial S=0$. It follows that $S=S_{\mathrm{N}}\left(\frac{\alpha}{\gamma} \delta+1\right)^{-1}$. Equation (10) then becomes

$$
F_{\mathrm{def}}=\frac{\alpha}{2} S_{\mathrm{N}}^{2} \delta+\left(\frac{\beta}{2}-\frac{\alpha^{2}}{2 \gamma} S_{\mathrm{N}}^{2}\right) \delta^{2}+\frac{\alpha^{3}}{2 \gamma^{2}} S_{\mathrm{N}}^{2} \delta^{3}+\mathcal{O}\left(\delta^{4}\right) .
$$

Putting all the pieces together we find equation (1) in the Results section.

Data availability. The authors declare that the relevant data supporting the findings of this study including raw data and MD simulations custom scripts are available from the corresponding authors on request.

\section{References}

1. Huygens, C. Extrait d'une lettre de M. Hugens de l'Académie Royale des Sciences à l'auteur de ce journal, touchant les phénomènes de l'eau purgée d'air, J. des Sçavants, 25 juillet 1672. Phil. Trans. 7, 5027 (1672).

2. Reynolds, O. Papers on Mechanical and Physical Subject Vol. I, 231 (Cambridge Univ. Press (1900-1903).

3. Reynolds, O. Papers on Mechanical and Physical Subject Vol. I, 394 (Cambridge Univ. Press (1900-1903).

4. Reynolds, O. An experimental investigation of the circumstances which determine whether the motion of water shall be direct or sinuous, and of the law of resistance in parallel channels. Proc. R. Soc. Lond. 174, 935 (1883)

5. Caupin, F. \& Herbert, E. Cavitation in water: a review. C. R. Physique 7, 1000 (2006).

6. Dowson, D. \& Taylor, C. M. Cavitation in bearings. Ann. Rev. Fluid Mech. 11, 35 (1979).

7. Zhang, J. \& Meng, Y. Direct observation of cavitation phenomenon and hydrodynamic lubrication analysis of textured surfaces. Tribol. Lett. 46, 147 (2012).

8. Arndt, R. E. A. Cavitation in fluid machinery and hydraulic structures. Ann. Rev. Fluid Mech. 13, 273 (1981).

9. Escaler, X., Egusquiza, E., Farhat, M., Avellan, F. \& Coussirat, M. Detection of cavitation in hydraulic turbines. Mech. Sys. Sig. Process 20, 983 (2006).

10. Gong, C. \& Hart, D. P. Ultrasound induced cavitation and sonochemical yields. J. Acoust. Soc. Am. 104, 2675 (1998).

11. Shchukin, D. G. \& Möhwald, H. Sonochemical nanosynthesis at the engineered interface of a cavitation microbubble. Phys. Chem. Chem. Phys. 8, 3496 (2006).

12. Hosangadi, A. \& Ahuja, V. Numerical study of cavitation in cryogenic fluids. Trans. ASME-I - J. Fluids Eng. 127, 267 (2005).

13. Son, Y. \& Migler, K. B. Cavitation of polyethylene during extrusion processing instabilities. J. Polym. Sci. Polym. Phys. 40, 2791 (2002).

14. Holbrook, N. M., Burns, M. J. \& Field, C. B. Negative xylem pressures in plantsa test of the balancing pressure technique. Science 270, 1193 (1995).

15. Kawchuk, G. N. et al. Real-time visualization of joint cavitation. PLoS ONE 10, e0119470 (2015).

16. Lim, W. L., Low, Y. T. \& Foo, W. L. Cavitation phenomena in mechanical heart valves: the role of squeeze flow velocity and contact area on cavitation initiation between two impinging rods. J. Biomech. 36, 1269 (2003).

17. Versluis, M., Schmitz, B., von der Heydt, A. \& Lohse, D. How snapping shrimp snap: through cavitating bubbles. Science 289, 2114 (2000).

18. Trevena, D. H. Cavitation and the generation of tension in liquids. J. Phys. D 17, 2139 (1984).

19. Debenedetti, P. G. Metastable Liquids: Concepts and Principles (Princeton University Press, 1996).

20. Debenedetti, P. G. Physics of water: stretched to the limit. Nature Physics 9, 7 (2013).

21. Baidakov, V. G., Bobrov, K. S. \& Teterin, A. S. Cavitation and crystallization in a metastable Lennard-Jones liquid at negative pressures and low temperatures. J. Chem. Phys. 135, 054512 (2011).

22. Baidakov, V. G. \& Bobrov, K. S. Spontaneous cavitation in a Lennard-Jones liquid at negative pressures. J. Chem. Phys. 140, 184506 (2014). 
23. Shen, V. K. \& Debenedetti, P. G. A computational study of homogeneous liquid-vapor nucleation in the Lennard-Jones fluid. J. Chem. Phys. 111, 3581 (1999).

24. Rasmussen, C. J., Gor, G. Y. \& Neimark, A. V. Monte Carlo simulation of cavitation in pores with nonwetting defects. Langmuir 28, 4702 (2012).

25. Meadley, S. L. \& Escobedo, F. A. Thermodynamics and kinetics of bubble nucleation: Simulation methodology. J. Chem. Phys. 137, 074109 (2012).

26. Oxtoby, D. W. \& Evans, R. Nonclassical nucleation theory for the gas-liquid transition. J. Chem. Phys. 89, 7521 (1988).

27. Zeng, X. C. \& Oxtoby, D. W. Gas-liquid nucleation in Lennard-Jones fluids J. Chem. Phys. 94, 4472 (1991).

28. Uline, M. J. \& Corti, D. S. Activated instability of homogeneous bubble nucleation and growth. Phys. Rev. Lett. 99, 076102 (2007).

29. Torabi, K. \& Corti, D. S. Molecular simulation study of cavity-generated instabilities in the superheated Lennard-Jones liquid. J. Chem. Phys. 133, 134505 (2010).

30. Chandrasekhar, S. Liquid Crystals (Cambridge University Press, 1992).

31. de Gennes, P. G. \& Prost, J. The Physics of Liquid Crystals (Oxford Science Publications, 1993).

32. Oswald, P. \& Pieranski, P. Nematic and Cholesteric Liquid Crystals: Concepts and Physical Properties Illustrated by Experiments (CRC Press, 2005).

33. Makke, A., Perez, M., Rottler, J., Lame, O. \& Barrat, J.-L. Predictors of cavitation in glassy polymers under tensile strain: a coarse-grained molecular dynamics investigation. Macromol. Theory Sim. 20, 826 (2011).

34. Toepperwein, G. N. \& de Pablo, J. J. Cavitation and crazing in rod-containing nanocomposites. Macromolecules 44, 5498 (2011).

35. Needham, J. Order and Life (MIT Press, 1936).

36. Collings, P. J. Liquid Crystals, Natures Delicate Phase of Matter (Princeton University Press, 1990).

37. Gray, G. in Chemistry Session: Molecular Self-Assembly in Science and Life (British Association for the Advancement of Science, 1993).

38. Knight, D. \& Feng, D. in Chemistry Session: Molecular Self-Assembly in Science and Life (British Association for the Advancement of Science, 1993).

39. Kruse, K., Joanny, J., Jülicher, F., Prost, J. \& Sekimoto, K. Generic theory of active polar gels: a paradigm for cytoskeletal dynamics. Eur. Phys. J. E 16, 5 (2005).

40. Furukawa, R., Kundra, R. \& Fechheimer, M. Formation of liquid crystals from actin filaments. Biochemistry 32, 12346 (1993).

41. Käs, J. et al. F-actin, a model polymer for semiflexible chains in dilute, semidilute, and liquid crystalline solutions. Biophys. J. 70, 609 (1996).

42. Vorreuther, R., Corleis, R., Klotz, T., Bernards, P. \& Engelmann, U. Investigative urology: impact of shock wave pattern and cavitation bubble size on tissue damage during ureteroscopic electrohydraulic lithotripsy. J. Urol. 153, 849 (1995).

43. Zhong, P., Tong, H. L., Cocks, F. H., Pearle, M. S. \& Preminger, G. M. Transient cavitation and acoustic emission produced by different laser lithotripters. J. Endourol. 12, 371 (1998).

44. Sengupta, A., Herminghaus, S. \& Bahr, C. Liquid crystal microfluidics: surface, elastic and viscous interactions at microscales. Liq. Cryst. Rev. 2, 73 (2014).

45. Manjuladevi, V., Pratibha, R. \& Madhusudana, N. Y. Phase transitions in liquid crystals under negative pressures. Phys. Rev. Lett. 88, 055701 (2002).

46. Luckhurst, G. R. On the creation of director disorder in nematic liquid crystals. Thin Solid Films 509, 36 (2006).

47. Deschamps, J., Trusler, J. P. M. \& Jackson, G. Vapor pressure and density of thermotropic liquid crystals: MBBA, $5 \mathrm{CB}$, and novel fluorinated mesogens. J. Phys. Chem. B 112, 3918 (2008)

48. Sengupta, A., Schulz, B., Ouskova, E. \& Bahr, C. Functionalization of microfluidic devices for investigation of liquid crystal flows. Microfluid. Nanofluid. 13, 941 (2012).

49. Sengupta, A., Pieper, C., Enderlein, J., Bahr, C. \& Herminghaus, S. Flow of a nematogen past a cylindrical micro-pillar. Soft Matter 9, 1937 (2013).

50. Gannon, M. G. J. \& Faber, T. E. The surface tension of nematic liquid crystals. Philos. Mag. A 37, 117 (1978).

51. Kasten, H. \& Strobl, G. Nematic wetting at the free surface of 4-cyano- $4^{\prime}-\mathrm{n}-$ alkyl-biphenyls. J. Chem. Phys. 103, 6768 (1995).

52. Khullar, S., Zhou, C. \& Feng, J. J. Dynamic evolution of topological defects around drops and bubbles rising in a nematic liquid crystal. Phys. Rev. Lett. 99, 237802 (2007)

53. Smela, E. \& Martinez-Miranda, L. J. Effect of substrate preparation on smectic liquid crystal alignment: a structural study. J. Appl. Phys. 73, 3299 (1993).

54. Huisman, W. J. et al. Layering of a liquid metal in contact with a hard wall. Nature 390, 379 (1997)

55. Cheng, L., Fenter, P., Nagy, K. L., Schlegel, M. L. \& Sturchio, N. C. Molecularscale density oscillations in water adjacent to a mica surface. Phys. Rev. Lett. 87, 156103 (2001)
56. Mishra, C. \& Peles, Y. Cavitation in flow through a micro-orifice inside a silicon microchannel. Phys. Fluids 17, 013601 (2005).

57. Mishra, C. \& Peles, Y. Flow visualization of cavitating flows through a rectangular slot micro-orifice ingrained in a microchannel. Phys. Fluids 17 113602 (2005).

58. Mishra, C. \& Peles, Y. An experimental investigation of hydrodynamic cavitation in micro-venturis. Phys. Fluids 18, 103603 (2006).

59. Sengupta, A. Topological Microfluidics: nematic liquid crystals and nematic colloids in microfluidic environment (Springer International Publishing, 2013).

60. Lee, J. N., Park, C. \& Whitesides, G. M. Solvent compatibility of poly (dimethylsiloxane)-based microfluidic devices. Anal. Chem. 75, 6544 (2003).

61. Kleman, M. \& Laverntovich, O. D. Soft Matter Physics: An Introduction (Springer Science \& Business Media, 2007).

62. Sengupta, A., Tkalec, U. \& Bahr, C. Nematic textures in microfluidic environment. Soft Matter 7, 6542 (2011).

63. Ilnytskyi, J. M. \& Wilson, M. R. A domain decomposition molecular dynamics program for the simulation of flexible molecules of spherically-symmetrical and nonspherical sites. II. Extension to NVT and NPT ensembles. Comput. Phys. Commun. 148, 43 (2002).

64. Stieger, T., Schoen, M. \& Mazza, M. G. Effects of flow on topological defects in a nematic liquid crystal near a colloid. J. Chem. Phys. 140, 054905 (2014).

65. Stieger, T., Püschel-Schlotthauer, S., Schoen, M. \& Mazza, M. G. Flow-induced deformation of closed disclination lines near a spherical colloid immersed in a nematic host phase. Mol. Phys. 114, 259 (2016).

66. Stoyanov, S. D. \& Groot, R. D. From molecular dynamics to hydrodynamics: a novel galilean invariant thermostat. J. Chem. Phys. 112, 114112 (2005).

67. Melle, M., Schlotthauer, S., Mazza, M. G., Klapp, S. H. L. \& Schoen, M. Defect topologies in a nematic liquid crystal near a patchy colloid. J. Chem. Phys. 136, 194703 (2012).

68. Tavoularis, S. Measurment in Fluid Mechanics (Cambridge University Press, 2005).

\section{Acknowledgements}

T.S. and M.S. gratefully acknowledge financial support by the Deutsche Forschungsgemeinschaft (DFG) within the framework of the 'International Graduate Reaserch Training Group' 1524, and M.G.M. thanks the Max Planck Society for funding. A.S. thanks Human Frontier Science Program Cross Disciplinary Fellowship (LT000993/2014-C) for supporting the research. We thank Carsten Krüger for suggestions and Stephan Herminghaus and Christian Bahr for helpful discussions at different stages of this work.

\section{Author contributions}

M.S., M.G.M. and A.S. designed research. T.S. performed computer simulations. H.A. and A.S. performed experiments and analysed data. M.G.M. developed the theoretical arguments. T.S., M.S., M.G.M. and A.S. discussed the results and prepared the manuscript. M.G.M. and A.S. coordinated the research and provided advice for all parts of the work

\section{Additional information}

Supplementary Information accompanies this paper at http://www.nature.com/ naturecommunications

Competing interests: The authors declare no competing financial interests.

Reprints and permission information is available online at http://npg.nature.com/ reprintsandpermissions/

How to cite this article: Stieger, T. et al. Hydrodynamic cavitation in Stokes flow of anisotropic fluids. Nat. Commun. 8, 15550 doi: 10.1038/ncomms15550 (2017).

Publisher's note: Springer Nature remains neutral with regard to jurisdictional claims in published maps and institutional affiliations.

This work is licensed under a Creative Commons Attribution 4.0 International License. The images or other third party material in this article are included in the article's Creative Commons license, unless indicated otherwise in the credit line; if the material is not included under the Creative Commons license, users will need to obtain permission from the license holder to reproduce the material. To view a copy of this license, visit http://creativecommons.org/licenses/by/4.0/

(C) The Author(s) 2017 